\title{
Biomechanical characterization of swimmers with physical disabilities
}

\author{
Valdir Junior ${ }^{1,2,3}$, Alexandre Medeiros ${ }^{3}$, Kelly de Jesus ${ }^{4}$, Nuno Garrido ${ }^{5}$, Rui Corredeira ${ }^{6}$, \\ Daniel J. Daly ${ }^{7}$, Ricardo J. Fernandes ${ }^{1,2^{*}}$
}

\begin{abstract}
The evaluation of swimming technique is one of the main aspects to be considered in any training program, with biomechanics being an important source of knowledge. It was our objective to characterize the biomechanical parameters (SL and SF) relating them to the swimming velocity $(v)$ at different intensities and to analyze within each swimming stroke cycle the intra-cyclic velocity variation (IVV) in a group of motor disabled swimmers. Eight disabled male swimmers $(25.83 \pm 2.93$ years old, $72.45 \pm 9.26 \mathrm{~kg}$ body mass and $1.79 \pm 0.11 \mathrm{~m}$ of height) of the following functional classes: S6 $(\mathrm{n}=1)$, S8 $(\mathrm{n}=2)$ and S9 $(\mathrm{n}=$ 5) participated in this study. Swimmers were evaluated in the kinematic parameters $v$, stroke frequency (SF) and stroke length (SL) along with an incremental protocol of $6 \times 200 \mathrm{~m}$ in the the crawl stroke. Data were registered in each step at the distances of 100 and $175 \mathrm{~m}$. With increasing velocity, the mean values of SL decreased while the mean values of SF increased. To achieve higher swimming velocities, swimmers compensated the lack of the propulsive segment increasing SF to increase swimming speed. For the mean values of IVV at $100 \mathrm{~m}$ distance, a decrease between the first and second levels, followed by a tendency to stabilize from the $2^{\text {nd }}$ to the $6^{\text {th }}$ level is presented. For the $175 \mathrm{~m}$ distance, there was a decrease in IVV with an increase in swimming velocity. Stroke frequency is directly related to the magnitude of IVV, which directly influences swimming performance.

Keywords: physical disability, biomechanical parameters, swimmers.
\end{abstract}

\section{INTRODUCTION}

The evaluation of the swimming technique is one of the main aspects to be considered in any training program (Fernandes et al., 2011) and one way of doing this is through biomechanical analysis. One of the main objectives of the biomechanical evaluation in competitive swimming (CS) is to characterize a certain swimming pattern aiming to enhance its efficiency (Barbosa et al., 2008), therefore allowing the swimmer to access higher levels of sports performance. Regarding swimming efficiency, one of the major concerns among biomechanics researchers in CS is the study of the mechanical factors that influence the kinematics of the center of mass, particularly the relationship between swimming speed $(v)$, stroke frequency (SF) and stroke length (SL) with competitive performance. This performance is directly related to the ability of the swimmer to coordinate a complex series of body movements aiming to maximize propulsion and minimize hydrodynamic drag. For each swim cycle of the crawl stroke, the swimmer must alternate the action of the upper limbs (UL), maintaining a horizontal and lateral streamlined position, and continuously and alternately execute the lower limb (LL) flutter kick (Osborough et al., 2009).

\footnotetext{
Manuscript received at April 23 ${ }^{\text {th }} 2016$; Accepted at October $8^{\text {th }} 2018$

${ }^{1}$ Centre of research, Education, Innovation and Intervention in Sport, Faculty of Sport, University of Porto, Portugal

${ }^{2}$ Porto Biomechanics Laboratory, University of Porto, Portugal

${ }^{3}$ Research Group in Biodynamic Human Movement, Institute of Physical Education and Sport, Federal University of Ceara, Brazil.

${ }^{4}$ Human Performance Studies Laboratory, Faculty of Physical Education and Physiotherapy, Federal University of Amazonas, Brazil.

${ }^{5}$ Research Center in Sports Sciences, Health Sciences and Human Development, CIDESD, Vila Real, Portugal

${ }^{6}$ Research Center in Physical Activity, Health and Leisure, Faculty of Sport, University of Porto

${ }^{7}$ Catholic University of Leuven, Belgium

* Corresponding author: R. Dr. Plácido da Costa 91, 4200-450, Porto, Portugal E-mail: ricfer@fade.up.pt
} 
Several studies characterize the efficiency of general biomechanical parameters (SF and SL), relating them to $v$ (Keskinen \& Komi, 1993; Toussaint et al., 2006). Already in the 1970s, Craig and Pendergast (1979) suggested that the crawl technique, when compared to other conventional swimming strokes, has the highest $\mathrm{SF}$ and SL values. Other studies describe that $v$ (by the combination of SF and SL) is highly individualized, depending on the swimmer's characteristics (Chollet et al., 1996; Kennedy et al., 1990). For Arellano et al. (1994), the success of swimmers in the crawl stroke is associated with longer SL, higher mean values of $v$ and greater stature. In a more recent study with adolescent swimmers, Laat et al. (2010) suggested that the variability in the $100 \mathrm{~m}$ crawl performance is explained in $\sim 90 \%$ by biomechanical factors.

Until the 1990s, despite the extensive literature on the biomechanical characterization of non-disabled swimmers, few studies have examined disabled swimmers. Daly et al. (1998), were the pioneers to evaluate the relationship between $v$, SF and SL in Paralympic swimmers, concluding that $v$ increases with SL. Pelayo et al. (1999) also evaluated general biomechanical parameters, relating them to $v$ and comparing high-level disabled with non-disabled swimmers, noting that there were no differences in SF between the two groups and that SL was related to a higher $v$. In crawl stroke, the hand and forearm are considered to be the largest propulsive surfaces, accounting for $\sim 85 \%$ of the total propulsion (Toussaint \& Beek, 1992). Thus the partial or complete absence of propulsive segments can cause changes in SF and SL (Prins \& Murata, 2008). Osborough et al. (2009) analyzed the relationship between $v, \mathrm{SF}$ and SL in crawl stroke and the anthropometric characteristics in swimmers with unilateral arm amputation. Authors concluded that due to the deprivation of a significant propulsive segment, $v$ combined with DF (in comparison to SL) is more determinant of swimming performance than SL which was found to have no relation with the anthropometric characteristics.

Another important and widely accepted criterion for the biomechanical studies in CS is the analysis of the intra-cyclic velocity variation (IVV) (Barbosa et al., 2006), which represents the swimmer's mechanical work to transpose the state of inertia (Nigg, 1983). Thus, to achieve a high mean swimming velocity, the propulsive forces produced by the swimmer will have to be higher than the opposed hydrodynamic drag forces (Villas-Boas et al., 2010). In this way, IVV can be considered a measure of propulsive efficiency, directly influencing swimmer's performance (Kornecki \& Bober, 1978), and is frequently used to evaluate the biomechanical development and coordination in CS. Disabled swimmers may present irregular SF and SL according to the absence or lack of mobility of one or several propulsive segments and may interfere with IVV. Therefore, the evaluation of IVV is of paramount importance in this population.

Considering the paucity of literature regarding swimmers with physical disabilities and the fact that the evaluation of the overall biomechanical parameters (SF and SL) related to $v$ and IVV may provide meaningful answers to a better understanding and development to performance in adapted swimming, the present study aimed to characterize the general biomechanical parameters (SF and SL), relating them to $v$ and IVV, at different swimming intensities. Thus, it was hypothesized that: 1) the methodology adopted allows to determine the general biomechanical parameters (SF and SL) relating them to $v$ in CS; 2 ) the SF and SL parameters change over an incremental intermittent protocol; and 3) there is a relationship between $v$ and IVV in CS over the incremental protocol.

\section{METHOD}

\section{Participants}

Eight male disabled swimmers $(25.83 \pm 2.93$ years old, $72.45 \pm 9.26 \mathrm{~kg}$ body weight and $1.79 \pm$ $0.11 \mathrm{~m}$ tall) registered in the Portuguese Swimming Federation participated in the present study. Subjects were classified according to the International Paralympic Committee in the following functional classes: S6 $(n=1)$, S8 $(n=$ $2)$ and $S 9(n=5)$. All swimmers were previously informed about the experimental protocol and had given their written consent to participate (in the case of underage the same was requested to 
the legal tutor). All subjects were familiar with the experimental testing procedures.

\section{Procedures}

The experimental sessions took place in a 25 $\mathrm{m}$ indoor heated swimming pool $\left(27^{\circ} \mathrm{C}\right)$, with a depth of $1.90 \mathrm{~m}$ and relative humidity of $85 \%$. Swimmers were weighed (using a bio-impedance scale InBody R20; Biospace Co, Ltd., Seoul, Korea) and measured (with a conventional tape). Then performed an intermittent incremental protocol of $6 \times 200 \mathrm{~m}$ crawl, with 30s of interval and increments of $0.05 \mathrm{~m} . \mathrm{s}^{-1}$ between each repetition of $200 \mathrm{~m}$ (Fernandes et al., 2003; Figueiredo et al., 2013). The velocity of the last step was established according to the best time of the moment of each swimmer at $400 \mathrm{~m}$ crawl, and the speed of the previous levels was calculated subtracting $0.05 \mathrm{~m} . \mathrm{s}^{-1}$ at the velocity of the final $200 \mathrm{~m}$ (Fernandes et al., 2003). During the protocol, a system of intermittent lights placed at the bottom of the swimming pool and distant 1.5 m (Pacer2Swim, KulzerTec, Aveiro, Portugal) was used, helping swimmers to maintain the predefined swimming velocity.

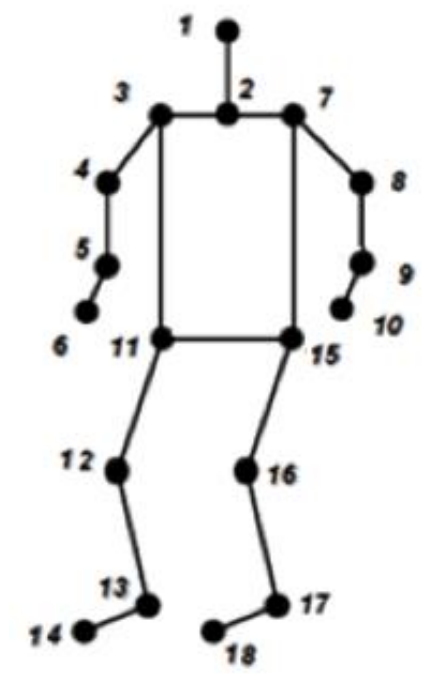

Figure 1. Diagram of the joint points marked on the swimmer (based on the model of Zatsiorsky et al., 1990 adapted by de Leva, 1996).

All subjects were marked with black ink and/or black tape on 18 anatomical points (right and left side of the body): acromion, lateral humeral epicondyle, ulna styloid process, femoral trochanter (external surface), lateral femoral epicondyle, lateral malleolus, distal phalanx and hallux, while the head apex was marked with a black tape on the swimming cap. Reflective markers were used to improve image visualization, subsequent digitalization and reconstruction in three dimensions (3D), using the 18 reference points as represented in Figure 1 (instead of the 21 points, commonly used and proposed by Zatsiorsky et al. al., 1990). For 3D reconstruction, the mass percentages and positions of the body segments were used (Zatsiorsky et al., 1990; Table 1).

\section{Table 1}

Values extracted from the model of reconstruction of the center-of-mass (de Leva, 1996; Zatsiorsky et al., 1990, adapted by de Leva, 1996)

\begin{tabular}{lcc}
\hline & Mass (\%) & $\begin{array}{c}\text { Longitudinal position of the } \\
\text { center of mass (\%) }\end{array}$ \\
Head & 6.94 & 59.76 \\
Right arm & 2.71 & 57.72 \\
Right forearm & 1.62 & 45.74 \\
Right hand & 0.61 & 79.00 \\
Left arm & 2.71 & 57.72 \\
Left forearm & 1.62 & 45.74 \\
Left hand & 0.61 & 79.00 \\
Trunk & 43.46 & 44.86 \\
Right thigh & 14.16 & 40.95 \\
Right leg & 4.33 & 44.59 \\
Right foot & 1.37 & 44.15 \\
Left thigh & 14.16 & 40.95 \\
Left leg & 4.33 & 44.59 \\
Left foot & 1.37 & 44.15 \\
\hline
\end{tabular}

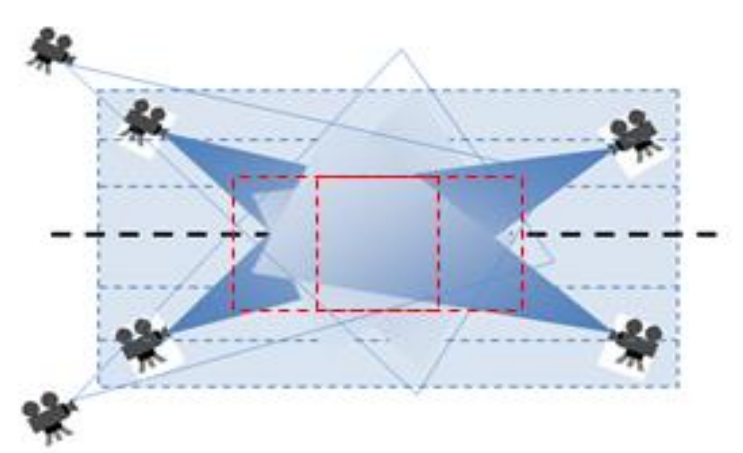

Figure 2. Illustration of the positioning of the underwater and surface cameras and calibration structure in the swimming pool.

Six video cameras (Sony ${ }^{\circledR}$ HDR CX160E, Tokyo, Japan) were used for image acquisition, operating at a frequency of $50 \mathrm{~Hz}$, with an electronic shutter speed of $1 / 250$. Four underwater cameras, protected by waterproof boxes (Sony ${ }^{\circledR}$ SPK-CXB waterproof box, Tokyo, 
Japan), were kept at $0.95 \mathrm{~m}$ below the water surface and positioned at 5.00 and $0.60 \mathrm{~m}$ from the frontal and side walls, respectively, and two aerial cameras which were maintained in a support at a height of $2.50 \mathrm{~m}$ and positioned at 2.10 and $1.10 \mathrm{~m}$ from the frontal and side walls, respectively, of the swimming pool (Figure 2).
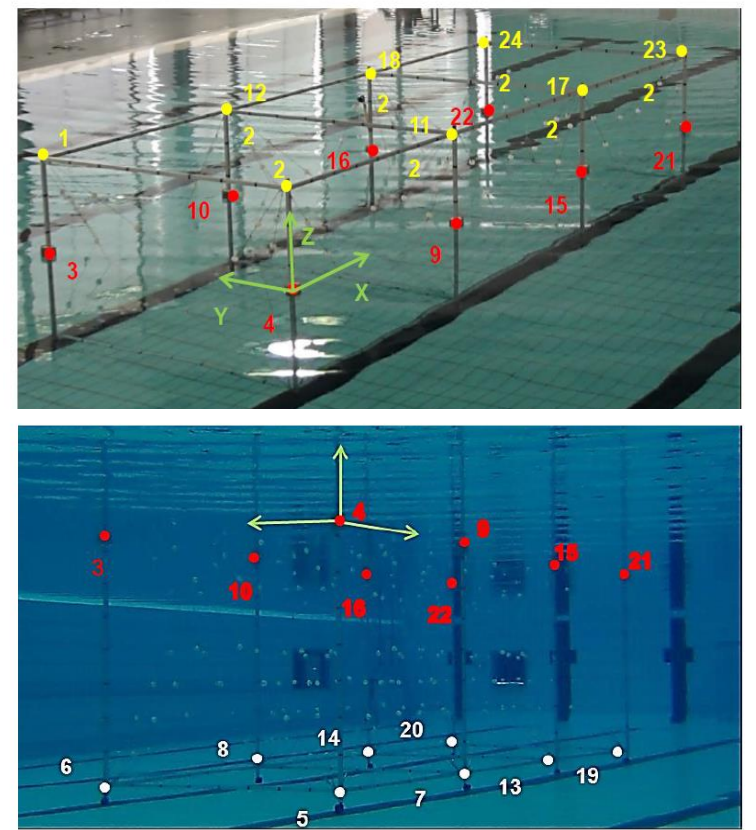

Figure 3. Aerial and underwater view (upper and lower panel, respectively) of the calibration space frame, showing the 24 calibration points of the Ariel Performance Analysis System.

The images of the six cameras were independently recorded, and the swimmers monitored as they passed through the previously calibrated space using a calibration space frame $6.0 \mathrm{~m}$ long, $2.0 \mathrm{~m}$ wide and $2.5 \mathrm{~m}$ high (de Jesus et al., 2014; Figure 3). The synchronization of the images was performed using a pair of LEDs, visible by all cameras, fixed to the calibration volume both under and at the surface of the water. For kinematic analysis, videos of two cycles of the crawl stroke (at 100 and 175m) were captured from each step of the incremental protocol. Videos were analyzed using the Ariel Performance Analysis System (APAS - Ariel Dynamics, San Diego, USA) at a frequency of 50 HZ. The 3D reconstruction of the upper limb action was performed using the Direct Linear Transformation (DLT) procedure.

\section{Statistical analysis}

The descriptive statistics were used to calculate the means and respective standard deviations, and the data were analyzed later using magnitude-based inference (Cumming, 2013; Hopkins et al., 2009). Differences in standardized means and respective confidence intervals of $95 \%$ were used to evaluate possible differences in SF, SL, and IVV between protocol steps and distances (100 and $175 \mathrm{~m}$ ). To calculate the magnitude of the effect, the following scale was used: $0-0.2$ trivial, $>0.2$ - 0.6 small, $>0.6-1.2$ moderate, $>$ $1.2-2.0$ large, and $>2.0$ very large (Hopkins et al., 2009) being considered values of effect sizes above small (positive or negative, Hopkins et al., 2009). All analyzes were performed using the Exploratory Software for Confidence Intervals (Cumming, 2013).

\section{RESULTS}

Table 2 presents the mean values and respective standard deviations of the kinematic parameters SF, SL and IVV at the distances of 100 and $175 \mathrm{~m}$ of each step of the incremental protocol.

Table 2

Mean and respective standard deviations of the kinematic parameters (stroke frequency and stroke length) and intra-cyclic velocity variation in 100 and $175 \mathrm{~m}$ of each step of the incremental protocol of $6 \times 200 \mathrm{~m} \mathrm{crawl}$

\begin{tabular}{ccccccc}
\hline Steps & $\begin{array}{c}\text { Stroke } \\
\text { length } \\
(\mathrm{m})\end{array}$ & $\begin{array}{c}\text { Stroke } \\
\text { frequency } \\
(\mathrm{Hz})\end{array}$ & $\begin{array}{c}\text { Intra-cyclic } \\
\text { velocity } \\
\text { variation }\end{array}$ & $\begin{array}{c}\text { Stroke } \\
\text { length } \\
(\mathrm{m})\end{array}$ & $\begin{array}{c}\text { Stroke } \\
\text { frequency } \\
(\mathrm{Hz})\end{array}$ & $\begin{array}{c}\text { Intra-cyclic } \\
\text { velocity } \\
\text { variation }\end{array}$ \\
\hline $1^{\text {st }}$ & $1.75 \pm 0.27$ & $0.47 \pm 0.11$ & $0.27 \pm 0.14$ & $2.00 \pm 0.25$ & $0.47 \pm 0.06$ & $0.27 \pm 0.24$ \\
$2^{\text {nd }}$ & $1.68 \pm 0.28$ & $0.51 \pm 0.09$ & $0.24 \pm 0.18$ & $1.84 \pm 0.25$ & $0.51 \pm 0.08$ & $0.27 \pm 0.24$ \\
$3^{\text {rd }}$ & $1.62 \pm 0.33$ & $0.54 \pm 0.10$ & $0.26 \pm 0.17$ & $1.76 \pm 0.23$ & $0.54 \pm 0.10$ & $0.22 \pm 0.16$ \\
$4^{\text {th }}$ & $1.60 \pm 0.34$ & $0.58 \pm 0.08$ & $0.27 \pm 0.22$ & $1.78 \pm 0.24$ & $0.60 \pm 0.05$ & $0.26 \pm 0.28$ \\
$5^{\text {th }}$ & $1.56 \pm 0.35$ & $0.61 \pm 0.09$ & $0.27 \pm 0.22$ & $1.66 \pm 0.32$ & $0.63 \pm 0.04$ & $0.21 \pm 0.20$ \\
$6^{\text {th }}$ & $1.48 \pm 0.36$ & $0.65 \pm 0.10$ & $0.24 \pm 0.16$ & $1.57 \pm 0.29$ & $0.72 \pm 0.06$ & $0.22 \pm 0.17$ \\
\hline
\end{tabular}


Table 3

Standardized mean differences, confidence intervals and effect size of the comparisons between incremental protocol levels at 100 and $175 \mathrm{~m}$ for (stroke length, stroke frequency and intra-cyclic velocity variation)

\begin{tabular}{|c|c|c|c|c|c|c|}
\hline Levels & $\begin{array}{c}\text { Stroke } \\
\text { length }(\mathrm{m})\end{array}$ & $\begin{array}{c}\text { Stroke } \\
\text { frequency }(\mathrm{Hz})\end{array}$ & $\begin{array}{c}\text { Intra-cyclic velocity } \\
\text { variation }\end{array}$ & $\begin{array}{c}\text { Stroke } \\
\text { length }(\mathrm{m})\end{array}$ & $\begin{array}{c}\text { Stroke } \\
\text { frequency }(\mathrm{Hz})\end{array}$ & $\begin{array}{c}\text { Intra-cyclic velocity } \\
\text { variation }\end{array}$ \\
\hline & & $100 \mathrm{~m}$ & & & $175 \mathrm{~m}$ & \\
\hline \multirow{2}{*}{$1^{\text {st }} \times 2^{\text {nd }}$} & $-0.22[-1.35 ; 0.90]$ & $0.33[-0.70 ; 1.36]$ & $-0.16[-1.39 ; 1.08]$ & $-0.49[-1.58 ; 0.61]$ & $0.50[-0.74 ; 1.73]$ & $0.01[-1.09 ; 1.11]$ \\
\hline & Small & Small & Trivial & Small & Small & Trivial \\
\hline \multirow{2}{*}{$1^{\text {st }} \times 3^{\text {rd }}$} & $-0.39[-1.62 ; 0.85]$ & $0.54[-0.52 ; 1.61]$ & $-0.09[-1.31 ; 1.13]$ & $-0.72[-1.77 ; 0.34]$ & $0.86[-0.60 ; 2.31]$ & $-0.16[-1.11 ; 0.80]$ \\
\hline & Small & small & Trivial & Moderate & Moderate & Trivial \\
\hline \multirow{2}{*}{$1^{\text {st }} \times 4^{\text {th }}$} & $-0.44[-1.69 ; 0.81]$ & $0.83[-0.15 ; 1.82]$ & $-0.04[-1.46 ; 1.39]$ & $-0.68[-1.77 ; 0.40]$ & $1.57[0.59 ; 2.55]$ & $-0.01[-1.21 ; 1.19]$ \\
\hline & Small & Moderate & Trivial & Moderate & Large & Trivial \\
\hline \multirow{2}{*}{$1^{\text {st }} \times 5^{\text {th }}$} & $-0.56[-1.83 ; 0.70]$ & $1.08[0.06 ; 2.09]$ & $-0.42[-1.36 ; 0.51]$ & $-1.03[-2.29 ; 0.24]$ & $1.97[1.04 ; 2.90]$ & $-0.17[-1.19 ; 0.84]$ \\
\hline & Small & Moderate & Small & Moderate & Large & Trivial \\
\hline \multirow{2}{*}{$1^{\text {st }} \times 6^{\text {th }}$} & $-0.80[-2.10 ; 0.50]$ & $1.35[0.27 ; 2.44]$ & $-0.16[-1.32 ; 1.00]$ & $-1.32[-2.52 ;-0.12]$ & $2.94[1.86 ; 4.03]$ & $-0.14[-1.11 ; 0.82]$ \\
\hline & Moderate & large & Trivial & large & Very large & Trivial \\
\hline \multirow{2}{*}{$2^{\text {nd }} \times 3^{\text {rd }}$} & $-0.16[-1.36 ; 1.04]$ & $0.25[-0.89,1.39]$ & $0.06[-1.03 ; 1.15]$ & $-0.23[-1.29 ; 0.83]$ & $0.29[-0.96,1.54]$ & $-0.17[-1.12 ; 0.79]$ \\
\hline & Trivial & Small & Trivial & Small & Small & Trivial \\
\hline \multirow{2}{*}{$2^{\text {nd }} \times 4^{\text {th }}$} & $-0.21[-1.42 ; 1.01]$ & $0.58[-0.46 ; 1.63]$ & $0.10[-1.12 ; 1.32]$ & $-0.20[-1.29 ; 0.90]$ & $0.87[-0.06 ; 1.81]$ & $-0.02[-1.22 ; 1.18]$ \\
\hline & Small & small & Trivial & Trivial & Moderate & Trivial \\
\hline \multirow{2}{*}{$2^{\text {nd }} \times 5^{\text {th }}$} & $-0.33[-1.56 ; 0.91]$ & $0.86[-0.21 ; 1.94]$ & $0.10[-1.12 ; 1.32]$ & $-0.55[-1.82 ; 0.73]$ & $1.19[0.29 ; 2.10]$ & $-0.19[-1.20 ; 0.83]$ \\
\hline & Small & Moderate & Trivial & Small & Large & Trivial \\
\hline \multirow{2}{*}{$2^{\text {nd }} \times 6^{\text {th }}$} & $-0.55[-1.82 ; 0.71]$ & $1.18[0.01 ; 2.36]$ & $0.00[-1.05 ; 1.05]$ & $-0.85[-2.05 ; 0.36]$ & $1.99[0.99 ; 2.98]$ & $-0.15[-1.12 ; 0.81]$ \\
\hline & small & large & Trivial & Moderate & Large & Trivial \\
\hline \multirow{2}{*}{$3^{\text {rd }} \times 4^{\text {th }}$} & - $0.04[-1.15 ; 1.07]$ & $0.31[-0.71 ; 1.33]$ & $0.04[-1.20 ; 1.28]$ & $0.04[-1.10 ; 1.17]$ & $0.46[-0.45 ; 1.37]$ & $0.22[-1.42 ; 1.85]$ \\
\hline & Trivial & Small & Trivial & Trivial & Small & Small \\
\hline \multirow{2}{*}{$3^{\text {rd }} \times 5^{\text {th }}$} & $-0.14[-1.27 ; 0.98]$ & $0.57[-0.47 ; 1.62]$ & $0.04[-1.20 ; 1.28]$ & $-0.34[-1.68 ; 1.01]$ & $0.72[-0.18 ; 1.62]$ & $-0.02[-1.26 ; 1.21]$ \\
\hline & Trivial & Small & Trivial & Small & Moderate & Trivial \\
\hline \multirow{2}{*}{$3^{\text {rd }} \times 6^{\text {th }}$} & $-0.33[-1.49 ; 0.82]$ & $0.87[-0.26 ; 2.00]$ & $-0.06[-1.12 ; 1.00]$ & $-0.66[-1.92 ; 0.60]$ & $1.35[0.41,2.29]$ & $0.02[-1.13 ; 1.18]$ \\
\hline & Small & Moderate & Trivial & Moderate & Large & Trivial \\
\hline \multirow{2}{*}{$4^{\text {th }} \times 5^{\text {th }}$} & $-0.10[-0.21 ; 1.01]$ & $0.31[-0.82 ; 1.44]$ & $0.00[-1.08 ; 1.08]$ & $-0.35[-1.64 ; 0.94]$ & $0.52[-0.47 ; 1.50]$ & $-0.14[-1.09 ; 0.81]$ \\
\hline & Trivial & small & Trivial & Small & Small & Trivial \\
\hline \multirow{2}{*}{$4^{\text {th }} \times 6^{\text {th }}$} & $-0.29[-1.42 ; 0.85]$ & $0.66[-0.58 ; 1.90]$ & $-0.08[-1.05 ; 0.89]$ & $-0.66[-1.88 ; 0.56]$ & $1.78[0.52 ; 3.04]$ & $-0.11[-1.05 ; 0.82]$ \\
\hline & Small & Moderate & Trivial & Moderate & Large & Trivial \\
\hline \multirow{2}{*}{$5^{\text {th }} \times 6^{\text {th }}$} & $-0.18[-1.31 ; 0.94]$ & $0.33[-0.87 ; 1.54]$ & $-0.08[-1.05 ; 0.89]$ & $-0.23[-1.28 ; 0.82]$ & $1.61[0.10 ; 3.11]$ & $0.04[-1.00 ; 1.08]$ \\
\hline & Trivial & Small & Trivial & Small & Large & Trivial \\
\hline
\end{tabular}




$$
\begin{aligned}
& 1^{\text {st }} \operatorname{step}(100 \mathrm{~m}) \times 1^{\text {st }} \operatorname{step}(175 \mathrm{~m}) \\
& 2^{\text {nd }} \operatorname{step}(100 \mathrm{~m}) \times 2^{\text {nd }} \operatorname{step}(175 \mathrm{~m}) \\
& 3^{\text {rd }} \operatorname{step}(100 \mathrm{~m}) \times 3^{\text {rd }} \operatorname{step}(175 \mathrm{~m}) \\
& 4^{\text {th }} \operatorname{step}(100 \mathrm{~m}) \times 4^{\text {th }} \operatorname{step}(175 \mathrm{~m}) \\
& 5^{\text {th }} \operatorname{step}(100 \mathrm{~m}) \times 5^{\text {th }} \operatorname{step}(175 \mathrm{~m}) \\
& 6^{\text {th }} \operatorname{step}(100 \mathrm{~m}) \times 6^{\text {th }} \operatorname{step}(175 \mathrm{~m})
\end{aligned}
$$

$1^{\text {st }} \operatorname{step}(100 \mathrm{~m}) \times 1^{\text {st }} \operatorname{step}(175 \mathrm{~m})$

$2^{\text {nd }}$ step $(100 \mathrm{~m}) \times 2^{\text {nd }}$ step $(175 \mathrm{~m})$

$3^{\text {rd }} \operatorname{step}(100 \mathrm{~m}) \times 3^{\text {rd }}$ step $(175 \mathrm{~m})$

$4^{\text {th }} \operatorname{step}(100 \mathrm{~m}) \times 4^{\text {th }}$ step $(175 \mathrm{~m})$

$5^{\text {th }} \operatorname{step}(100 \mathrm{~m}) \times 5^{\text {th }}$ step $(175 \mathrm{~m})$

$6^{\text {th }} \operatorname{step}(100 \mathrm{~m}) \times 6^{\text {th }}$ step $(175 \mathrm{~m})$

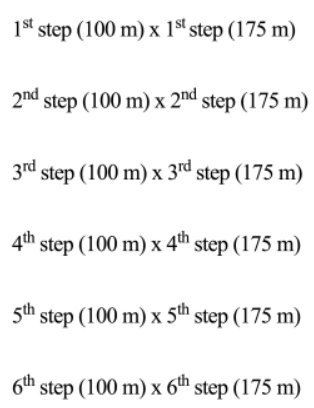

a) Stroke length

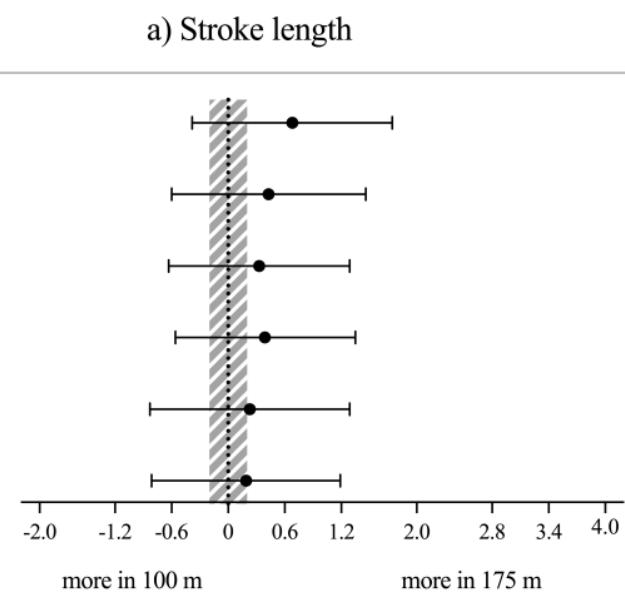

b) Stroke frequency

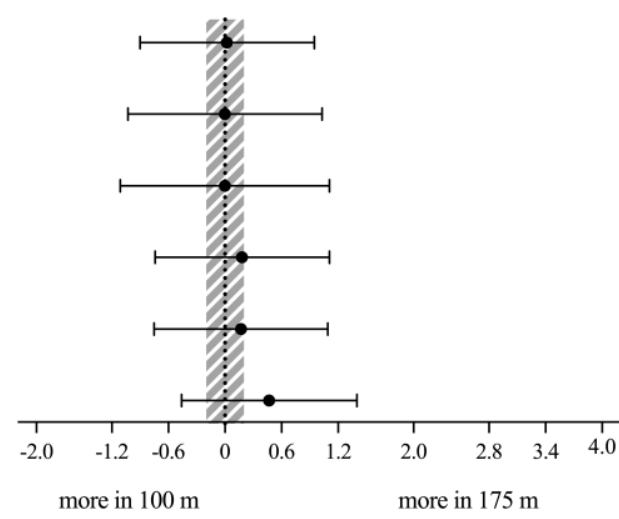

$\begin{array}{cc}0,02[-0,90 ; 0,95] & \text { Trivial } \\ 0,00[-1,03 ; 1,03] & \text { Trivial } \\ 0,00[-1,11 ; 1,11] & \text { Trivial } \\ 0,18[-0,74 ; 1,11] & \text { Trivial } \\ 0,17[-0,75 ; 1,09] & \text { Trivial } \\ 0,47[-0,46 ; 1,40] & \text { Trivial }\end{array}$

c) Intra-cyclic velocity variation

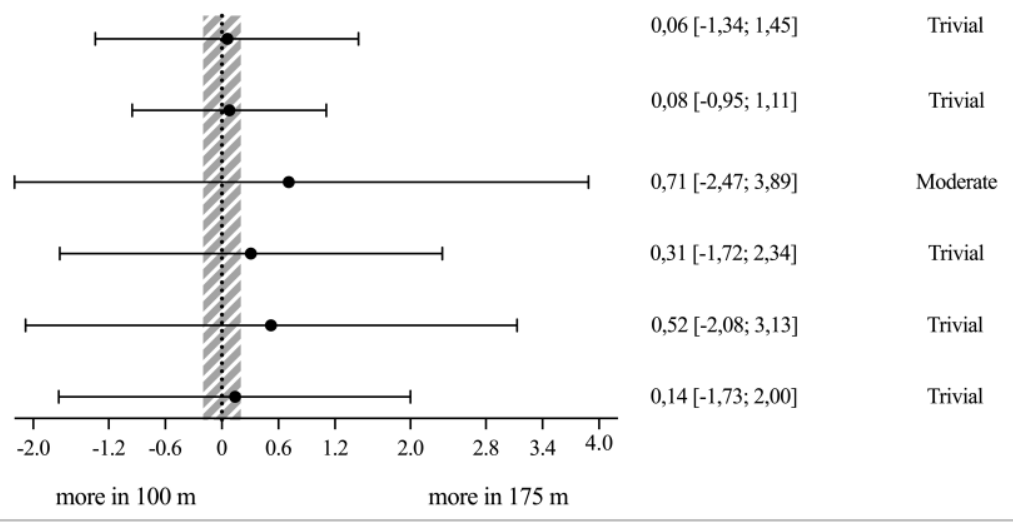

$\begin{array}{cc}\text { SMD }[\mathrm{IC} 95 \%] & \text { Effect size } \\ 0,68[-0,38 ; 1,74] & \text { Moderate } \\ 0,43[-0,60 ; 1,46] & \text { Trivial } \\ 0,33[-0,63 ; 1,29] & \text { Trivial } \\ 0,39[-0,56 ; 1,35] & \text { Trivial } \\ 0,23[-0,83 ; 1,29] & \text { Trivial } \\ 0,19[-0,81 ; 1,19] & \text { Trivial } \\ \end{array}$

\section{Trivial \\ Trivial \\ rivial \\ Trivial \\ Trivial \\ Trivial}

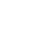


compared to the first, second and fourth levels and in the fourth level when compared with the first one at the distance of $175 \mathrm{~m}$.

Figure 4 presents the standardized mean differences of, confidence intervals and effect size of the comparisons between 100 and $175 \mathrm{~m}$ at each step of the incremental protocol for the kinematic parameters of the SF, SL, and IVV (panels a, b and c), showing trivial differences.

\section{DISCUSSION}

The objective of this study was to characterize the general biomechanical parameters in the crawl stroke, relating them to $v$ at the distances of 100 and $175 \mathrm{~m}$ from low to high swimming intensities in a group of disabled swimmers belonging to the functional classes between S6 and S9. Complementarily, it was also assessed the IVV in each stroke cycle. The main findings of the present study indicate that swimmers modify their general biomechanical parameters (SL and $\mathrm{SF}$ ) when velocity increases and also between distances of 100 and $175 \mathrm{~m}$ from each $200 \mathrm{~m}$ step. On the other hand, there was a tendency to stabilize IVV with increasing velocity along the protocol steps. This fact suggests an adaptation to the requirements related to the increasing swimming velocity, where swimmers change their technique, which is in agreement with the studies described in the literature.

In the present study, with increasing $v$ at each step, the values of the SL decrease and the values of SF increase at the distances of 100 and $175 \mathrm{~m}$ (corroborating the studies of Keskinen \& Komi, 1993, Osborough et al. 2009 and Seifert et al. 2004). The decreased of SL may be linked to the development of local muscle fatigue as suggested by Keskinen and Komi (1993). Moreover, it should be pointed out that asymmetry may also compromise the swimmer's technique (Dingley et al., 2014). In the current study it was found that in order to achieve higher velocities, swimmers with lower limb amputation need to increase the effort to maintain body alignment, and swimmers with upper limb amputation need to compensate for the lack of the propulsive segment (Prins \& Murata, 2008) thus, increasing SF to concomitantly increase $v$ (Osborough et al., 2009).
In this study it was also observed that the SL values, within each step, are higher at the distance of $175 \mathrm{~m}$ when compared to $100 \mathrm{~m}$ at all steps, while the SF values remain constant from the first to the third level, increasing from the fourth to the sixth step at the distance of $175 \mathrm{~m}$ in comparison to $100 \mathrm{~m}$. This increase can be explained by the dependence that disabled swimmers have on the SF to get higher $v$ (Figueiredo et al., 2013; Satkunskiene et al., 2005) and also to the increasing fatigue over time (Dingley et al., 2014).

Substantially higher values were observed in the sixth step when compared to the second one for $\mathrm{SF}$ at $100 \mathrm{~m}$. At the distance of $175 \mathrm{~m}$, SF values showed substantially higher values in the sixth step when compared to the other steps (first to fifth). In the fifth step when compared to the first, second and fourth ones; and in the fourth step when compared to the first one, suggesting that during more exhaustive $v$, disabled swimmers, as reported for non-disabled swimmers (Alberty et al., 2008; Chatard et al., 1990; Craig \& Pendergast, 1979), limit the decrease of $v$ by increasing SF, compensating for the decline in SL.

The IVV is an important and widely accepted criterion for swimming biomechanical study (Barbosa et al., 2006) and is the best indicator of the swimmer's technical ability (Vilas-Boas et al., 2010). In this study, the values found for IVV are higher than those found in the CS literature, namely for swimmers with Down Syndrome (0.17) (Marques-Aleixo et al., 2013). The findings indicate that IVV values did not change significantly over the six steps at the distance of $100 \mathrm{~m}(0.24 \geq 0.27)$. There was a tendency to stabilize IVV from the second to the sixth step, suggesting that swimmers were able to adapt their swimming technique to minimize IVV. These values agree with those found by Seifert et al. (2010). At the distance of $175 \mathrm{~m}$, a decrease in IVV was observed with increasing velocity, which may be related to the time decrease between propulsive actions due to increased fatigue (Alberty et al., 2008; Ribeiro et al. 2013; Soares et al., 2010). It is worth noting that Fatigue causes a decrease in $v, \mathrm{SF}$ and SL (Craig \& 
Pendergast, 1979; Keskinen \& Komi, 1993; Marinho et al., 2006).

For the maintenance of $v$ it was observed a progressive increase in the values of the SF with a concomitant decrease of the SL throughout the incremental protocol. These adaptations corroborate with the findings of Alberty et al. (2008), Craig et al. (1985), Dekerle et al. (2005), Fernandes et al., (2010) and Wakayoshi et al. (1996), and could be attributed to the appearance of fatigue. In a fatigue condition, swimmers will gradually be unable to produce the necessary propulsive actions to overcome drag (Alberty et al., 2008), which justifies the decrease of IVV. IVV values found in this study are close to the findings by Payton and Wilcox (2006) who also analyzed only the propulsive actions of the upper limbs.

Even though we recognize the limitations of this study, which are related to the sample size and the low number of functional classes (S6, S7, S8, and S9) studied, which limits the generalization of the results for all the population of disabled swimmers, the presented results can bring essential insights for the biomechanical analysis of the crawl stroke for this group of swimmers. Future studies with larger sample size, more substantial variability of disabilities and a broader range of functional classes are needed to determine the factors that contribute to the performance of disabled swimmers in competitive swimming.

\section{CONCLUSION}

With the present study, it was possible to conclude that $\mathrm{SF}$ is directly related to the increase in $v$ and IVV magnitude and that IVV directly influences the performance of swimmers. In order to increase their swimming velocity, disabled swimmers, as non-disabled swimmers, should decrease IVV. Coaches and swimmers should create strategies in their training programs aiming to maintain body alignment in water, increase SF and decrease IVV to achieve better results in Competitive Swimming.

\section{Acknowledgments:}

Nothing to declare.

\section{Conflict of interests:}

Nothing to declare.

\section{Funding:}

Nothing to declare.

\section{REFERENCES}

Alberty, M., Potdevin, F., Dekerle, J., Pelayo, P., Gorge, P., \& Sidney, M. (2008). Changes in swimming technique during time to exhaustion at freely chosen and controlled stroke rates. Journal of Sports Sciences, 26(11), 1191-1200.

Arellano, R., Brown, P., Cappaert, J., \& Nelson, R. (1994). Analysis of 50-, 100-, and 200-m freestyle swimmers at the 1992 Olympic Games. Journal of Applied Biomechanics, 10, 189-199.

Barbosa, T., Fernandes, R., Keskinen, K., Colaço, P., Cardoso, C., Silva, J., \& Vilas- Boas, J. P. (2006). Evaluation of the energy expenditure in competitive swimming strokes. Training $\mathcal{E}$ Testing.

Barbosa, T., Fernandes, R., Morouco, P., \& Vilas-Boas, J. P. (2008). Predicting the intra-cyclic variation of the velocity of the centre of mass from segmental velocities in butterfly stroke: A pilot study. Journal of Sport Science and Medicine, 7, 201209.

Chatard, C., Collomp, C., Maglischo, E., \& Maglischo, C. (1990). Swimming skill and stroking characteristics of front crawl swimmers. International Journal of Sports Medicine, 11 (2), 156161.

Chollet, D., Pelayo, P., \& Tourney, C. (1996). Comparative analysis of $100 \mathrm{~m}$ and $200 \mathrm{~m}$ events in the four strokes in top level swimmers. Journal of Human Movement Studies 31, 25-37.

Craig, A., \& Pendergast, D. (1979). Relationship of stroke rate, distance per stroke, and velocity in competitive swimming. Medicine and Science in Sports 11(3), 278-283.

Craig, A., Skehan, P., J., P., \& Boomer, W. (1985). Velocity, stroke rate and distance per stroke during elite swimming competition. Medicine and Science in Sports Exercise, 17, 625-634.

Cumming, G. (2013). The new statistics: Estimation for better research. Retrieved from www.thenewstatistics.com.

Daly, D., Malone, L., Vanlandewijck, Y., \& Steadward, R. (1998). Analysis of the mean's 100m freestyle at the 1996 Atlanta Paralympic Games. Biomchanics and Medicine in Swimming.

de Jesus, K., Guidetti, L., de Jesus, K., Vilas-Boas, J. P., Baldari, C., \& Fernandes, R. J. (2014). Which ate the best VO2 sampling intervals to characterize low to severe swimming intensities? International Journal of Sports Medicine, 35(12), 1030-1036.

de Leva, P. (1996). Adjustments to ZatsiorskySeluyanov's segment inertia parameters. Journal of Biomechanics, 29(9), 1223-1230. 
Dekerle, J., Nesi, X., Lefevre, T., Depretz, S., Sidney, M., Marchand, F. H., \& Pelayo, P. (2005). Stroking parameters in front crawl swimming and maximal lactate steady state speed. International Journal of Sports Medicine, 26(1), 53-58.

Dingley, A. A., Pyne, D., \& Burkett, B. (2014). Dryland bilateral hand-force production and swimming performance in paralympic swimmers. International Journal of Sports Medicine, 35(11), 949953.

Fernandes, R., Cardoso, C., Soares, S., Ascenção, A., Colaço, P., \& Vilas-Boas, J. P. (2003). Time Limit and VO2 Slow Component at Intensities corresponding to VO2max in Swimmers. International Journal of Sports Medicine, 24, 576-581.

Fernandes, R. J., Sousa, M., Machado, L., \& Vilas-Boas, J. P. (2011). Step length and individual anaerobic threshold assessment in swimming. International Journal of Sports Medicine, 32(12), 940-946.

Fernandes, R. J., Sousa, M., Pinheiro, A., Vilar, S., Colaço, P., \& Vilas-Boas, J. P. (2010). Assessment of individual anaerobic threshold and stroking parameters in swimmers aged 10-11 years. European Journal of Sport Science, 10(5), 311-317.

Figueiredo, P., Toussaint, H. M., Vilas-Boas, J. P., \& Fernandes, R. J. (2013). Relation between efficiency and energy cost with coordination in aquatic locomotion. European Journal of Applied Physiology, 113(3), 651-659.

Hopkins, W., Marshall, S., Batterham, A., \& Hanin, J. (2009). Progressive statistics for studies in sports medicine and exercise science. Medicine and Science in Sports and Exercise, 41 (1), 3-12.

Kennedy, P., Brown, P., Chengalur, S., \& Nelson, R. (1990). Analysis of male and female Olympic swimmers in the 100-meter events. International Journal of Sport Biomechanics, 6(2), 187-197.

Keskinen, K., \& Komi, P. V. (1993). Strokin characteristics of front crawl swmming during exercise. Journal of Applied Biomechanics, 9, 219226.

Kornecki, S., \& Bober, T. (1978). Extreme velocities of a swimming cycle as a technique criterion. In B. Eriksson \& B. Furberg (Eds.), Swimming medicine IV (pp. 402-407). Baltimore: University Park Press.

Laat, E., Jurimae, J., Maestu, J., Purge, P., Ramson, R., Haljaste, K., Keskinen, K., Rodriguez, F., \& Jurimae, J. (2010). Physiological, biomechanical and anthropometrical predictors of sprint swimming performance in adolescent swimmers. Journal of Sports Science and Medicine, 9, 398-404.

Marinho, D., Vilas-Boas, J. P., Keskinen, K., Rodriguez, F., Soares, S., Carmo, C., Vilar, S., \& Fernandes, R. J. (2006). The behaviour of kinematic parameters during a time to exhaustion test at VO2max in elite swimmers. Journal of human Movement Studies, 51(51), 1.

Marques-Aleixo, I., Querido, A., Figueiredo, P., VilasBoas, J. P., \& Corredeira, R. (2013). Intracyclic velocity variation and arm coordination assessment in swimmers with down syndrome. Adpted Physical Activity Quarterly, 30(70-84).

Nigg, B. (1983). Selected methodology in biomechanics with respect to swimming. In A. Hollander, P. Huijing \& G. Groot (Eds.), Biomechanics and Medicine in Swimming (pp. 72-80): Champaign, Illinois: Human Kinetics.

Osborough, C., Payton, C., \& Daly, D. (2009). Relationships between the front crawl stroke parameters of competitive unilateral arm amputee swimmers, with selected anthropometric characteristics. Journal of Applied Biomechanics, 25, 304-312.

Payton, C., \& Wilcox, C. (2006). Intracyclic speed fluctuations of uni-lateral arm amputee front crawl swimmers. Revista Portuguesa de Ciências do Desporto, 6(1), 53-54.

Pelayo, P., Sidney, M., Moretto, P., Wille, F., \& Chollet, D. (1999). Stroking parameters in top level swimmers with a disability. Physical Fitness and Performance, 31 (12), 1839-1843.

Prins, J., \& Murata, N. (2008). Stroke Mechanics of Swimmers with Permanent Physical Palaestra, 24(1), 19-25.

Ribeiro, J., K., D. J., Figueiredo, P., Toussaint, H., Guidetti, L., Alves, F., Vilas-Boas, J. P., \& Fernandes, R. J. (2013). Biomechanical determinants of force production in front crawl swimming. Journal of Sports Medicine \& Physical Fitness, 53, 30-37.

Satkunskiene, D., Schega, L., Kunze, K., Birzinyte, K., \& Daly, D. (2005). Coordination in arm movements during crawl stroke in elite swimmers with a loco-motor disability. Human Movement Science, 24(1), 54-65.

Seifert, L., Chollet, D., \& Bardy, B. G. (2004). Effect of swimming velocity on arm coordination in the front crawl. A dynamic analysis. Journal of Sports Sciences, 22(7), 651-660.

Seifert, L., Toussaint, H. M., Alberty, M., Schnitzler, C., \& Chollet, D. (2010). Arm coordination, power, and swim efficiency in national and regional front crawl swimmers. Human Movement Science, 29(3), 426-439.

Soares, S., Aleixo, I., Machado, L., Fernandes, R. J., Maia, J., \& Vilas-Boas, J. P. (2010). Evaluation of force production and fatigue using an anaerobic test performed by differently matured swimmers In P. L. Kjendlie, R. Stallman \& J. Cabri (Eds.), Biomechanics and Medicine in Swimming XI (pp. 291293). Oslo, Norway Norwegian School of Sport Sciences

Toussaint, H., \& Beek, P. (1992). Biomechanics of competitive front crawl swimming. Sports Medicine, 13(1), 8-24.

Toussaint, H., Carol, A., Kranenborg, H., \& Truijens, M. (2006). Effect of fatigue on stroking characteristics in arm-only 100-m front-crawl race. Medicine and Science in Sports and Exercise, 38(9), 1635-1642.

Vilas-Boas, J. P., Fernandes, R., \& Barbosa, T. (2010). Intra-cycle velocity variations, swimming 
112 | V Junior, A Medeiros, K Jesus, N Garrido, R Corredeira, DJ Daly, RJ Fernandes

economy, performance and training in swimming. In L. Seifert, D. Chollet \& I. Mujika (Eds.), World book of swimming: from science to performance: Nova Science Publishers.

Wakayoshi, K., D'Acquisto, J., Cappaert, J. M., \& Troup, J. P. (1996). Relationship between metabolic parameters and stroking technique characteristics in front crawl. In J. P. Troup, A. P. Hollander, D. Strasse, S. W. Trappe, J. M.
Cappaert \& T. A. Trappe (Eds.), Biomechanics and medicine in swimming VII (pp. 152-158). London: Chapman \& Hall.

Zatsiorsky, V. M., Seluyanov, V. N., \& Chugunova, L. G. (1990). Methods of determining mass-inercial characteristics of human body segments. In G. Chemy \& S. Regirer (Eds.), Contemporary Problems of Biomechanics (pp. 272-291). Massachussets: CRC Press.

All content of Journal Motricidade is licensed under Creative Commons, except when
otherwise specified and in content retrieved from other bibliographic sources c) otherwise specified and in content retrieved from other bibliographic sources. 